\title{
Exploring the User Experiences of a Bird ID Mobile App Among College Students in Western India
}

\author{
Jayvijay V. Raol \\ West Virginia University, jraol@mix.wvu.edu
}

Follow this and additional works at: https://researchrepository.wvu.edu/etd

Part of the Educational Technology Commons, and the Science and Technology Studies Commons

\section{Recommended Citation}

Raol, Jayvijay V., "Exploring the User Experiences of a Bird ID Mobile App Among College Students in Western India" (2020). Graduate Theses, Dissertations, and Problem Reports. 7788.

https://researchrepository.wvu.edu/etd/7788

This Thesis is protected by copyright and/or related rights. It has been brought to you by the The Research Repository @ WVU with permission from the rights-holder(s). You are free to use this Thesis in any way that is permitted by the copyright and related rights legislation that applies to your use. For other uses you must obtain permission from the rights-holder(s) directly, unless additional rights are indicated by a Creative Commons license in the record and/ or on the work itself. This Thesis has been accepted for inclusion in WVU Graduate Theses, Dissertations, and Problem Reports collection by an authorized administrator of The Research Repository @ WVU. For more information, please contact researchrepository@mail.wvu.edu. 
Exploring the User Experiences of a Bird ID Mobile App Among College Students in Western India

Jayvijay Raol

Dissertation Submitted to the College of Education and Human Services

At the West Virginia University

In partial fulfillment of the requirements for the degree of Doctor of Education in Instructional Design \& Technology

\author{
Ugur Kale, Ph. D., Chair \\ Jiangmei Yuan, Ph. D. \\ Donald Trisel, Ph. D. \\ Abhik Roy, Ph. D. \\ Department of Learning Sciences and Human Development
}

\author{
Morgantown, West Virginia
}

2020

Keywords: User Experience, Mobile Applications, Instructional Affordances, Knowledge and Awareness, Human Computer Interaction, Qualitative Study.

Copyright 2020 Jayvijay Raol 


\begin{abstract}
Exploring the User Experiences of a Bird ID Mobile App Among College Students in Western India
\end{abstract}

Jayvijay Raol

This study explores user experiences of a sample of college age young adults from western India, with a bird ID mobile app. Mobile apps provide an attractive and interactive access to information and their reach and versatility can often bypass challenges of poor infrastructure and lack of access to sources for information. Studies that examine the influence of mobile apps on users' knowledge and awareness are still in their infancy. Mobile phones and mobile applications have become increasingly prevalent in the educational setting, not only as technology for instructional delivery, but also as a means of improving user experience when accessing information. Researchers have been studying the usability features and end- user experiences from mobile applications for innovative design improvements (Tang, Abraham, Stamp \& Greaves, 2015). Despite its potential to impact learning and user attitude, very limited research exists on how passive interactions with certain content specific mobile applications may increase users' knowledge and awareness on issues of contemporary importance to our society. Therefore, given the ever-growing reach of mobile devices and applications, the researcher is interested in exploring if a mobile app that allows users to identify the birds of western India, influences their knowledge and awareness of birds around them. This study will provide insight on how mobile apps may be designed to provide unique experiences leading to awareness and change in conservation knowledge and perception. 


\section{Content}

Literature 1

Enhancing Knowledge and Awareness about Conservation ....................................... 2

Studies on Knowledge and Awareness in Health and Wellness.................................. 3

Pilot Study with the Merlin Bird ID app and Resulting Observations ........................ 4

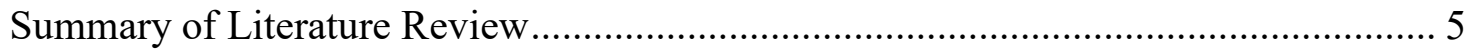

Research Focus and Research Questions ............................................................... 6

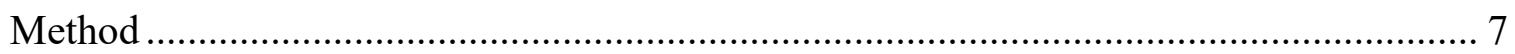

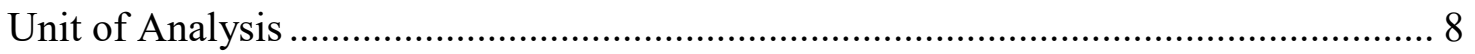

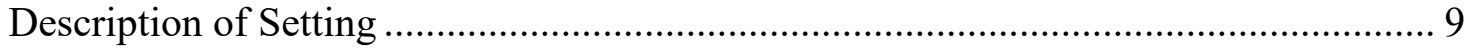

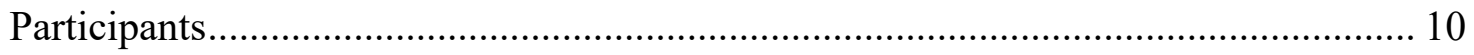

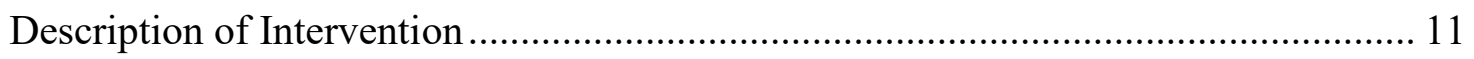

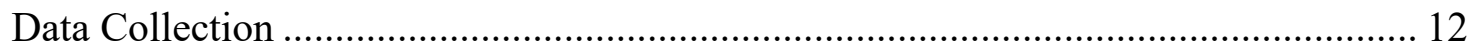

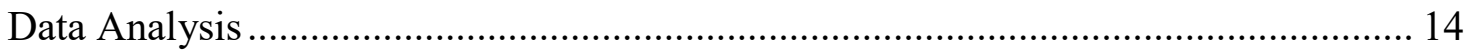

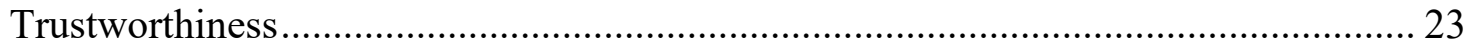

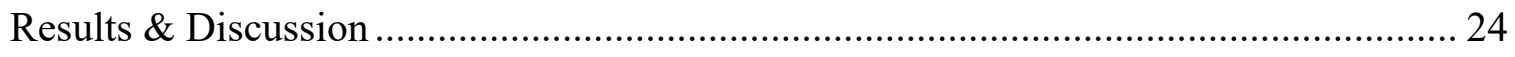

RQ1. Experiences of Users with the Hobby Bird ID Mobile App ............................ 24

RQ2. How Do Experiences with the App Influence Knowledge and Awareness of

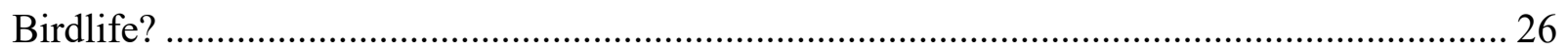

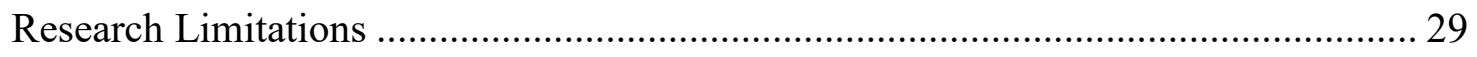




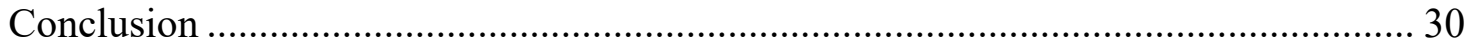

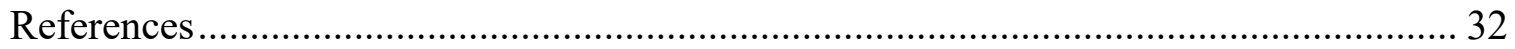

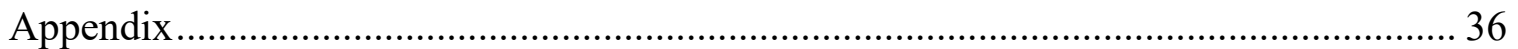




\section{Literature}

Numerous studies in the community and health sciences field have examined user knowledge and user perceptions using quantitative surveys. Usability studies on mobile applications have also been conducted using surveys and usage statistics. Limited research currently exists on the subjective aspects of the experiences and the broader influences from certain content specific mobile applications. The purpose of this study is to explore the user experiences of college students from Western Indian with an interactive bird ID app and examine if the interactive bird ID app influences users' knowledge and awareness of the local birdlife in in that region.

Environmental and wildlife awareness is a very pressing contemporary issue in India, with an urgent need for participatory governance from local community members. A general lack of awareness about the ecological functions and the balance of healthy ecosystems is a primary challenge to conservation efforts in India (Ambastha, Hussain, \& Badola, 2007). A report on the State of Indian Birds (Jain 2020), which was collaboratively prepared by various governmental and non-governmental agencies, has provided a startling statistic, claiming a decline in $79 \%$ of Indian bird species. The rich birdlife in India is faced with multi-pronged challenges, which include rampant deforestation, increased exposure to industrial and residual chemicals (Cuthbert et al, 2016), competition from domesticated species and the effects of global warming. The western Indian region of Saurashtra has always been a favorite destination for bird lovers and ornithologists from around the world for its rich local and migratory birdlife. In this semi-arid landscape, there is also a striking decrease in bird populations and researchers have stated that 
there is an urgent need to raise awareness (Green et al., 2004) as one of the primary steps to combat this drastic decline.

\section{Enhancing Knowledge and Awareness about Conservation}

Jhala and Giles (1991) have argued that the foremost step towards conservation is related to encouraging public support and education. A study in the geographically close North-West Frontier province has demonstrated a lack of awareness and a low level of participation in protected area management by the local citizens, but a high degree of willingness in the communities to participate in conservation activities. Paik and his colleagues (2009) used an online information system in Korea to study how a digital system may be used to increase community involvement in bird identification, eventually leading to increased awareness and preservation of bird diversity. Heinen and Shrivastava (2009) conducted an analysis of conservation attitudes and awareness in a different region in India and suggest that participatory approaches coupled with greater efforts in education and awareness are especially needed to achieve conservation and development goals. These studies clearly point to a need for improving awareness and knowledge, thereby paving a pathway towards participatory approaches in conservation.

Given this set of challenges, and the inherent instructional capabilities of mobile applications, there exists a singular opportunity for the study of the effects of this 'digital conservation' exercise. The term 'digital conservation' is used by Arts, Van and Adams (2015), in an attempt to show how digital technology is changing nature conservation in increasingly profound ways, comprising of five pivotal dimensions: data on nature, data on people, data integration and analysis, communication and experience, and participatory governance. 
This study was conducted in the Saurashtra region of Western India. The interest in this geographic region and this general population is based upon several premises. As India makes rapid advances in the area of mobile and communication technologies, it is observed that the remotest areas in the Saurashtra region, while they may be lacking in the most basic infrastructure, power and living amenities, are mostly well connected to the internet and mobile data access. As mobile technology and applications increasingly find instructional relevance, there is a need for new studies to be conducted on their application to educate, to raise awareness and to change the attitude of people with regard to important contemporary issues of wildlife conservation, decreasing human-animal conflict and understanding of the adverse effects of irresponsible waste disposal. Moreover, studies conducted in Africa (Garrigan, 2014) have found that in developing countries, technology makes it possible to bypass poor infrastructure and connect remote areas with the tools and services that provide access to information.

\section{Studies on Knowledge and Awareness in Health and Wellness}

A study of several medical journals, clinical trial registries, and databases (Wieland et al., 2012) suggests that digital applications such as mobile apps, provide an attractive, self-delivered way to prompt and support users to change their behavior and attitudes by providing interactive access to educational information. The terms knowledge and awareness are commonly juxtaposed in research literature. For this study, knowledge is referenced as information that is acquired from authoritative external sources and that can therefore be regarded as factual in nature while awareness is referenced to mean familiarity, attentiveness or attitude (Trevethan, 2017). The medical and health science fields are replete with studies that measure changes in knowledge and awareness from interventions, and several instruments exist for such studies based upon varying types of research designs. Gustafsson and Borglin (2013), Nooshin et al., 
(2018). For instance, in a study on attitudes of participants who interacted with a website, Kim and Stout (2010) found that interacting with an informational website on health and well-being affected the attitude of participants with regards to people with mental illness. The results of this study showed that interactivity of web-based communication had significant positive effects on processing of mental illness-related information and on attitudinal dimensions of mental illness stigma.

\section{Pilot Study with the Merlin Bird ID app and Resulting Observations}

Although studies that examine the impact of mobile applications on users' knowledge and awareness beyond the health science field are limited, interactions with mobile applications may have potential benefits with regards to informing users and positively influencing their perceptions on several contemporary issues such as environmental conservation and wildlife protection. As a preliminary step, a small user-experience pilot study on the Merlin bird ID app developed by the Cornell Ornithology Lab, was conducted by the researcher using a constructivist framework among a small group of college age students in West Virginia with the focus of exploring their developing knowledge and awareness of birds. A broader, more subjective view of user-experience was referenced for conducting this study, as explained by Araz (2018). This pilot study revealed several interesting observations about how interacting with the app had the potential to lead the users to become more cognizant about birds around them. For instance, the users reported that after using the app several times, they were better able to identify common birds around them and knew more about the habits and characteristics of the birds that they had identified with the app. The results of this study were used to guide the design of a similar app with additional interactive and social functionality as well as a much larger database, to be used in western India for this proposed qualitative study. Specifically, the app, 
called Hobby, consists of a database of around 600 birds found in western India and allows users to identify birds that they see, by answering five simple questions. The app also provides users with details about the bird, including photographs, bird calls and information about the habitat, behavior and conservation status of the birds. Furthermore, the app provides users with a bird directory feature, that displays a listing of birds by category, and is interactive.

Due to the short time frame required to market, multiplatform capabilities and embrace of fast emerging digital developments, mobile applications are one of the fastest emerging, public use technologies (Vaupel, Taentzer, Gerlach, \& Gucker, 2018). Mobile apps have already become a powerful medium for awareness and training campaigns. Most studies however, on this use of mobile apps, are limited to quantitative usability analyses. Moreover, changes in specific subject knowledge and awareness from mobile interventions are generally measured using tests and or surveys that provide data for empirical analysis. The literature from the community and health sciences fields uses instruments like the Knowledge, Attitudes and Practices surveys while fields like marketing and communications generally use statistical data from prototypes and usage statistics. Although these methods may justify research on extremely focused criteria, they do not allow for a deeper understanding of influences on knowledge and perceptions. A qualitative case study therefore would allow for this form of a subjective exploration as suggested by Vredenburg (2002) in the comprehensive discussion on designing the total user experience at IBM.

\section{Summary of Literature Review}

There is sufficient evidence to support a need for raising knowledge and awareness about local ecology and wildlife in India, and studies support the use of technology as an intervention with the reach and technological affordances to fulfill this need. However, there is limited 
research on the use of specific mobile apps to influence user awareness and increase knowledge. As mobile data and smartphone access becomes increasingly affordable and widely and easily available to the people of India, studies that explore and investigate these connections become increasingly attractive in the realm of user experience, cognitive and behavioral and human computer interaction research arenas. A qualitative case study design would provide the opportunity for an in-depth exploration of user experiences and the users' own perceptions of the influence of a newly developed and comprehensive mobile app on their knowledge and awareness.

\section{Research Focus and Research Questions}

This proposed study aims to explore the user experiences with an informational bird ID mobile app and investigate if these experiences influence knowledge and awareness of local birds. This qualitative study stems from the researcher's interest in gaining a deeper understanding of several aspects of mobile app design and usage, including the instructional affordances of mobile apps, the possible use of informational mobile apps to raise awareness and understanding of behavioral aspects of user experiences with mobile software. Considering the researcher's interest in gaining an in-depth exploration of user-experiences (Garman, 1994), a subjective research approach has been selected. This will allow for a wider perspective on possible cognitive and behavioral influences and affordances from the use of certain types of mobile apps. Subjective user experience studies for mobile apps are still in their infancy, even though Roherer (2014) outlines qualitative user interviews as one of the top 5 most valuable user experience research methods. Thus, the research questions explored in the current study are:

- RQ1: What are the experiences of college age young adults from western India with the Hobby bird ID mobile app? 
- RQ2: How do these experiences influence their knowledge and awareness of local birdlife?

\section{Method}

This study followed a case study approach wherein a qualitative methodology was employed to increase interpretability, meaningfulness and rigor. This design was chosen to provide a deeper understanding of how the app was perceived by users and individual user accounts on the experiences and cognitive affordances of the app. Cresswell (1998) suggests that the context of the case involves situating the case within its setting, which may be physical, historical etc. For this study, the researcher particularly used an explanatory case study approach to focus primarily on user experience and any causal links or influence to knowledge and awareness. This type of case study is used when seeking to explain the presumed causal links in real-life interventions that are too complex for the survey or experimental strategies (Yin, 2003). User experience is and should be treated in a subjective context. A clear distinction needs to be made here between usability studies that are generally empirical and user experience studies that focus on deeper, subjective understanding. Yin further emphasizes that an important strength of case studies is the ability to undertake an investigation into a phenomenon in its context. Since the user-experiences of each individual with the mobile app were unique and based purely upon the individual's perspectives, needs, environment, interest and motivation, the researcher did not control or manipulate the user behavior or interaction in any way. The phases of the study, along with the procedures and products of each phase are outlined in Figure 1. 


\section{Figure 1}

Explanatory Case Study Design

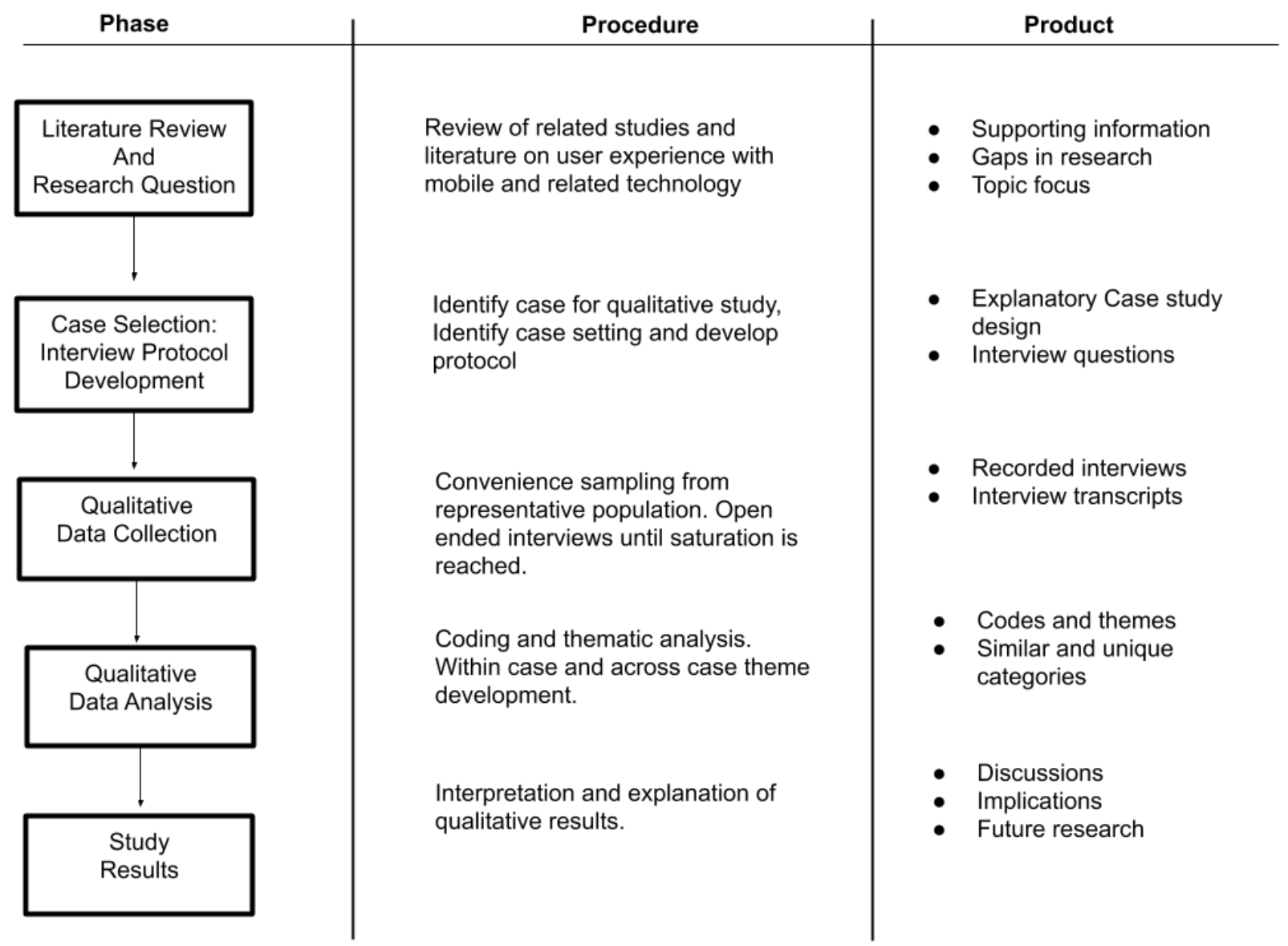

\section{Unit of Analysis}

Miles \& Huberman (1993, p. 25) define a case as "a phenomenon occurring in a bounded context" The case is, "in effect, your unit of analysis". Considering the unique experiences with the mobile app, juxtaposed alongside cultural and personal experiences of the users, it is, as the 'case', within the bounds of a specific age group and the specific environment of the western Indian region of Saurashtra, therefore provide the context for the case.

Each user was provided with the bird ID mobile app and explained the usage and functionality of the app. Interactions of the users with the app could vary, as stated above, based 
upon individual user's preferences, needs, interest and environment. The app also anonymously recorded specific limited usage data such as number of uses, date and time of use and specific bird identified. This data further assisted in making useful connections between user-experience and perceptions of the app with the frequency of usage. The qualitative semi-structured interviews were used to gather deeper and more meaningful stories from each individual's experience with the app and the perceptions of the users about if and how the app influenced their knowledge and awareness of local birds.

\section{Description of Setting}

A college age, young-adult population was of special interest because a recent study by Vserv (2013) has found that users between the age of 18-24 are the largest spectrum of smartphone users and data consumers in the overall mobile phone market in India. Moreover, data from Communications Today (2019) asserts that the state of Gujarat has an average consumption of $13 \mathrm{~GB}$ of mobile data per month, per connection, compared to the national average of $10 \mathrm{~GB}$ for the rest of the mobile users around India. This data provides useful insight justifying the access and availability of mobile data in the specific area of interest. Moreover, The Times of India (2019), India's leading newspaper, has published information stating that for every 100 smartphone users in the state of Gujarat, there are 104 connections. This statistic is also significantly higher than the national average for India. While specific age demographics are not available for mobile data users in the state of Gujarat, based upon the statistics listed above, it may be safely assumed that college age young adults form a sizeable spectrum of mobile data consumption for the state. 


\section{Participants}

Participants were selected from three different universities in Western India to justify an appropriate sample of college-age young adults from the general geographical area of interest. Participants were recruited with placement of ads introducing the focus of the research and a proper IRB protocol was followed throughout this process. To be eligible for participation, participants had to be currently enrolled college students, have a personal smartphone with a data connection, be regular users of mobile apps and be willing to participate in the study. A convenience sample was used, which was selected from institutions that showed interest in this study and offered support to collaborate. The researcher had previously discussed his research interests with department heads and administrators at some of the universities in the region of interest, and there had been a general offer of support and willingness for participation by these universities. Participants were chosen randomly from amongst the students of these institutions who met the minimum criteria mentioned above, to obtain a certain level of generalizability to the overall population. Apart from providing an overview of the study in the recruitment poster, participants who responded to the ad were given a more thorough explanation of the study along with an explanation of the bird ID app and how it functioned and were provided with responses to any questions that they may have had about the study. A sample size of $n=19$ was initially selected, with a distribution of 7, 6 and 6 participants from the three participating institutions. There were 6 female and 13 male participants with an age range between 18 and 22 years. All of the participants were enrolled in undergraduate programs at one of the participating institutions.

Stake (1995) cautions that case studies should be bound by a specific time and place to avoid excessively broad focus within a study and therefore a recruitment across a wider spectrum of institutions was not considered advisable by the researcher. All the participants were provided 
simultaneous access to the mobile and the data collection was also done within a timeframe that allowed each user to have approximately the same amount of exposure and access to the app, to generate a more unified set of perspectives. The participants from one of the institutions were also members of a college birding club $(n=7)$, while the participants from the remaining two institutions were not affiliated with any birding or nature related, institution sponsored activities $(n=12)$. Eventually, three of the participants, two from the institution with the birding club affiliation and one from a non-affiliated institution were unable to meet with the researcher for the scheduled interviews due to unforeseen circumstances, and therefore the researcher was able to interview 16 of the participants ( 4 female, 12 male with a distribution of 5,5,6 across the three institutions) at the end of the period allotted for the participants to use and experience the mobile app.

\section{Description of Intervention}

The Hobby Bird ID app has been specifically designed to serve as a mobile bird ID tool and directory of birds of Gujarat. There are currently 599 different birds identified by the Bombay Natural History Society in the state of Gujarat (Ganpule, 2016). Several academic and volunteer ornithology societies and programs supported the data collection for use with this mobile application. The app allows users to create an account, and browse details of all the birds in the region, sorted by categories. The app is highly interactive, and also allows users to identify birds from sightings, by allowing the users to answer five simple questions about the sighting. A deductive algorithm is used to derive the most likely birds based upon location, size, color and behavior of the bird, as provided by the user. Once identified, the user may choose to get additional information about the bird, including distribution, habitat, behavior and IUCN status. Bird calls are also included for several of the birds in the database, and the users have the option 
to save birds by sighting for future reference. Users may also share their bird sighting and information via social media platforms such as Facebook, Twitter or Whatsapp.

The app provides a simple user interface, packaged with a powerful cloud-based, data driven bird identification and multimedia functionalities. Data has been scientifically categorized and a deductive reasoning algorithm provides a high level of identification accuracy. The participants, after being familiarized with the mobile app, were asked to download and use and explore the app, for a period of 30 days, at their convenience. Each participant was assigned a unique identifier, such as user1, user2 and so on, and the participants were only referred by these identifiers throughout the study, to maintain anonymity.

\section{Data Collection}

Each participant was provided with an individual, pre-setup user account corresponding to their unique identifier, so that no personal data was needed to be entered or utilized in conjunction with the app. These pre-set user accounts allowed the researcher to study and analyze each users' bird identification history and usage statistics, which were made available to each user at the time of the interview, to serve as prompts and to assist with recall about specific times and experiences. Participants were scheduled for interviews at their respective campuses after approximately 21 days of access to the Hobby app. All individual interviews were scheduled in designated and private office settings within respective campuses where the participants were enrolled. Semi-structured interview questions, as outlined in Appendix A, were used to collect data, as they can provide reliable and comparable qualitative data and an in-depth narrative may be obtained (DiCicco-Bloom and Crabtree, 2006). Individual experiences might vary, and therefore the sample questions outlined, served as prompts to elucidate rich data about the participant's experiences and perceptions. The interviews were conducted in either English or 
Gujarati, which is the local language for that region, depending on the preference of the participant. The researcher is proficient in both languages and was able to translate all interviews to English during transcription, to allow uniform coding and categorization. Individual interviews were scheduled and conducted at the site of the participating institution where the student participant was enrolled. The primary goal of the interview was to explore unique user experiences with the app and the individual perceptions of users on personal cognitive affordances or influences during and after the use of the app. The questions for the interview were developed based upon the main research questions, followed a logical sequence and allowed for in-depth narratives.

The researcher took notes during the individual qualitative interviews, which were also recorded and transcribed for further analysis. As suggested by Marshall and Rossman (1995), the interviews provided rich narratives about personal experiences with the app as well as unique individual perceptions and perspectives. The researcher was also able to get immediate clarifications and a deeper explanation on several unique user experiences during the interview process. The researcher was able to conduct one on one interviews with 15 of the participants, and had to schedule a phone interview with one participant, who was unable to meet the researcher at a scheduled time, but did want to participate in the study.

The interview process itself provided for the first level of coding, and participants were notified that they may be contacted again for a follow-up interview, if the researcher discovered new themes emerging from successive interviews and would like to revisit certain parts of the interview. However, the individual semi-structured interviews allowed for sufficient clarity and quality of responses and therefore the need to re-interview did not arise in this case. Participants were also requested to participate in providing feedback during the data analysis, to improve 
interpretability of the data and two of the participants who were contacted during the data analysis, consented and provided their feedback.

\section{Data Analysis}

An explanatory single case study is particularly suitable for answering "how" and "why" questions and is also appropriate for generating and building theory in an area where little data or theory exists (Yin, 2003). It also enables the researcher to use "controlled opportunism" to permit a flexible response to new discoveries made while collecting new data (Einsenhardt, 1989). In the present case, in the absence of specific supporting research literature, the researcher was making a proposition about the users' experiences based upon a similar pilot study that was conducted by the researcher on a related, geographically separate population. The results of this pilot study suggested connections between the use of a bird ID app, and an increased interest and awareness in birds.

Within this case study approach, the researcher was able to observe some aspects of user behavior with the app such as the number of interactions, specific birds viewed and birds that were identified, via the usage statistics and history features. During the interviews, the researcher realized that one subset of the participants from a particular institution were all zoology majors within a Bachelor of Science program and were also actively engaged in a birding club affiliated with their institution. They informed the researcher that they were specially attracted to participating in this research because they felt that the research was connected to birding and ornithology. Since there was no assumption on the part of the researcher and no specific requirement for participants to have any prior interest, knowledge or association with birding or related activities, and all participants had been briefed about the purpose of this research at the time of recruitment, this specific subset of 5 participants provided an additional opportunity to 
observe some unique viewpoints, as well as an opportunity to see the differences in opinions of participants with varying prior knowledge and interest in birds.

The interviews yielded several perspectives and statements that were documented by the researcher during the interviews, and some preliminary in vivo codes were collected during the conversations with the participants. An inductive-deductive approach (Saldana, 2016) was used, since the researcher had made certain assumptions based upon literature review and the results of the pilot study but he also looked for new information during the data collection and subsequent analysis. Such an approach was deemed appropriate so that a researcher may carry with them what they know, but also listen to what is "new" in the data, as data is analyzed. The interview questions, while being directly related to the main research questions, also allowed for elaborate narrative and the researcher used probes where necessary as guides and to obtain rich and descriptive responses. Cresswell (2007) suggests beginning the process of "lean coding" with a short list of provisional codes (derived from the research questions) and expanding these into several categories which then combine into major themes. This specific coding format was followed by the researcher, beginning with in vivo codes related to the research questions, deriving code clusters or categories from these, and finally creating themes using the process of pattern coding.

The focus of this study includes both ontological as well as epistemological questions and therefore multiple coding techniques were utilized for analysis. Saldana (2016) suggests using In vivo coding as a sound method for ontological questions, while Pattern coding is more universal, and suitable for epistemological questions. In vivo coding derives from the words and phrases used by the participants (Straus, 1987), and therefore allows the researcher to capture the vividness of the user's individual experience in their own, unfiltered form. Most interviews 
lasted between 12 and 20 minutes and the researcher took notes while recording the interviews with prior permission from the participants. The researcher grouped specific statements and sentences from the interviews, that best reflected some of the pre-conceived categories that the researcher was interested in exploring. Table 1 provides an overview of one participant interview, with the in vivo codes noted down by the researcher in the right column.

Table 1

Sample participant interview with In vivo codes

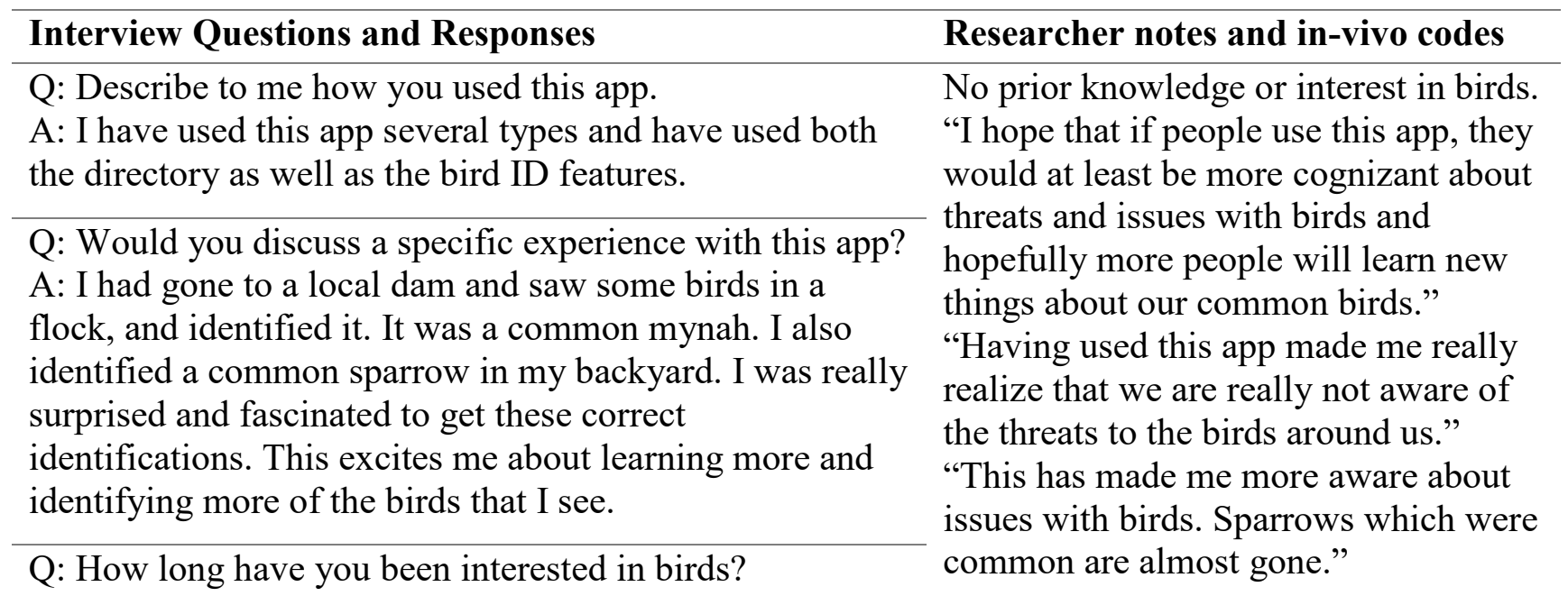

A: I have not been specifically interested in birds before this, and you can say that my knowledge of birds is very average or below.

Q: Can you recall any early experiences related to birds? A: I do not have any specific experiences related to birds that I can recall.

Q: Did you learn anything new about birds from this app?

Please discuss

A: Yes, having used this app made me really realize that we are really not aware of the threats to the birds around us. I even went to a bird rescue site to see more about what is happening to these birds and I attribute this curiosity to this app. 
Q: Would you say that having used this app, that you are more aware of threats or concerns to local birds? Please elaborate.

A: Yes, definitely, this has made me more aware about issues with birds. Sparrows which were common are almost gone. I hope that if people use this app, they would at least be more cognizant about threats and issues with birds and hopefully more people will learn new things about our common birds. And I feel that once people get these feelings about birds and the situation that we have, perhaps more people will want to learn and do more for conservation.

Analysis of in vivo codes allowed for categorization of outlined clusters and provided an opportunity for cross-examination between similarities and contrasts for the data from different participants. For instance, participants' spontaneous recall of names of specific birds that they had identified, interwoven within the narrative of their experiences with the app, were coded as "Recall". Similarly, expressions such as "I now realize", "I have become more sensitive to" and "I notice more birds", that were suggestive of a change in awareness, and were therefore significant to the researcher and thus duly noted down during the interviews. The Figure 2 below provides a snapshot of the concept map of the first round of in vivo codes collected during the interview and subsequent data analysis, and sub codes derived from these. 


\section{Figure 2}

\section{First cycle coding}
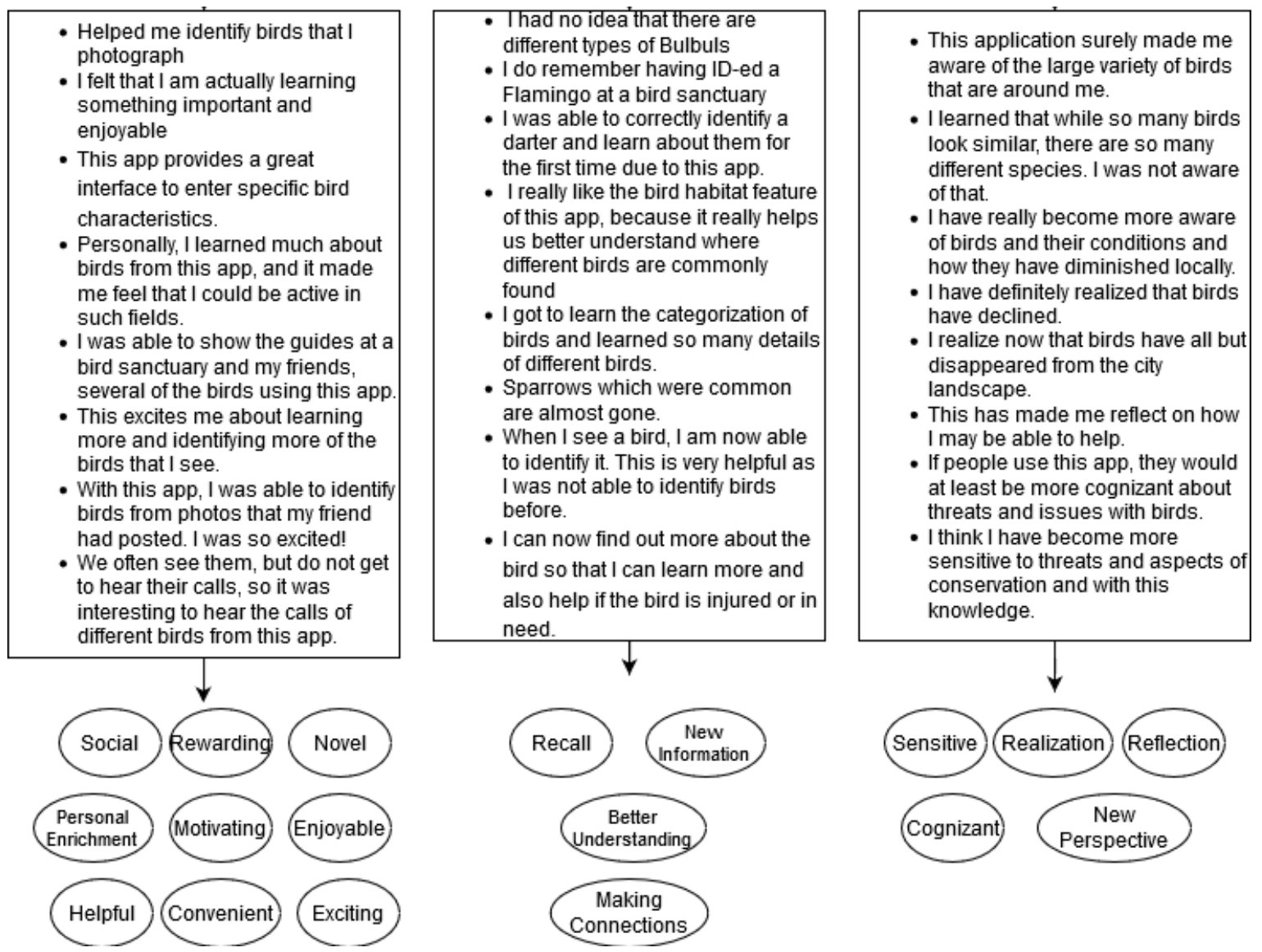

Note. Sample of in vivo codes collected during the interviews and the derivation of meaningful

codes within categories.

The frequency of the codes appearing in the interviews, that were identified within the code cluster, are provided in Table 2 below. 


\section{Table 2}

\section{Code frequency}

\begin{tabular}{ll}
\hline Codes & Frequency \\
\hline Social & 4 \\
Exciting & 5 \\
Rewarding & 2 \\
Novel & 3 \\
Personal Enrichment & 4 \\
Motivating & 3 \\
Helpful & 6 \\
Convenient & 7 \\
Enjoyable & 5 \\
Recall & 8 \\
New Information & 9 \\
Better Understanding & 9 \\
Making Connections & 8 \\
Sensitive & 2 \\
Realization & 5 \\
Reflection & 4 \\
Cognizant & 3 \\
New Perspective & 3
\end{tabular}

Additionally, the researcher was further able to categorize user perception of the app, based upon their prior knowledge and interest or lack of as discussed above. These subsets provided varying perspectives about whether the app could be used as a tool for general awareness among the target population. The codes derived from responses linked to individual perceptions of the app as a tool to raise awareness were observed to be strongly influenced by the participant's prior interest and knowledge, with those who had prior interest and knowledge providing more neutral responses, while those who did not have prior interest and had a limited knowledge of birds, strongly favoring such an app as a tool for general awareness on local birds. This was a new observation, which inductively emerged from the interview data and was influenced by factors (prior knowledge and interest in birds) that were not initially considered as a specific criterion during the planning of this study. Furthermore, initial data analysis also 
revealed that some of the participants did not use the bird ID feature within the app because of a perceived lack of bird sightings in their urban surroundings.

Pattern coding was utilized to further analyze the data, as Pattern coding is an appropriate second cycle coding method when the researcher wishes to search for rules, causes and explanations within the data (Saldana, 2016). This method of coding allowed the researcher to refine the dominant codes and simultaneously categorize data for differences and relationships. Saldana (2016) further suggests that pattern coding allows the use of tacit and intuitive senses to determine which data "look alike" and "feel alike" when grouping them together. This coding method helped the researcher to further categorize the in vivo code cluster groups to relevant themes such as 'Positive experiences' and 'Increased awareness'. The Figure 3 below displays a snapshot of the themes that emerged from the second cycle pattern coding during the data analyses.

\section{Figure 3}

Second cycle coding
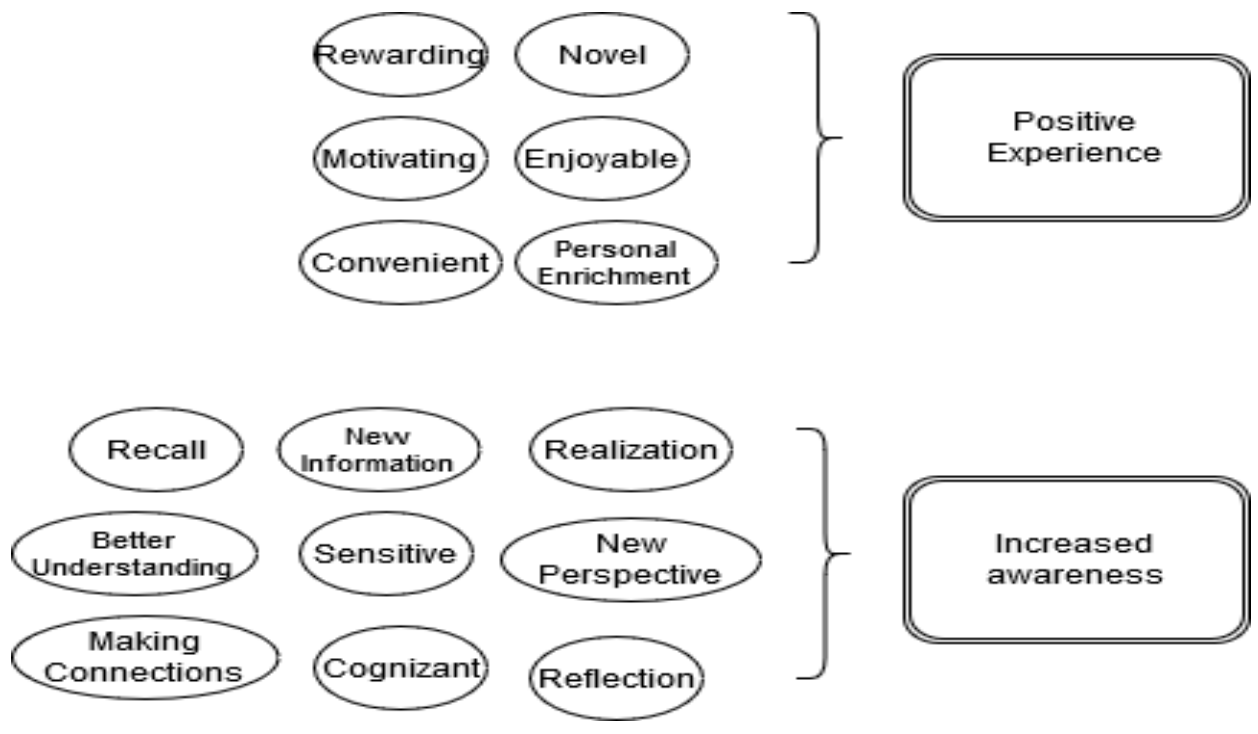

Note. Finding patterns within first cycle codes to create meaningful themes 
Moreover, as mentioned before, some of the participants were involved in activities within an extra-curricular birding club affiliated with their institution. These participants had some prior interest and knowledge about birds, and their perception of the bird app was as a guide for birding and bird watchers, while participants who had limited or no prior interest in birds and were not involved in birding related activities, perceived the app as a tool to become more aware about the variety and general condition of the birds in the region. This led to additional themes related to app perception. Responses from these sub groups also suggested that participants who had previous interest and knowledge perceived the app as a tool to improve their knowledge and use as reference, but did not necessarily always see it as a tool for raising awareness. However, the participants who had limited prior knowledge of birds, all strongly felt that the app made them feel more aware of the birds around them and made them realize the decline in bird populations. They also spent more time reading about the information provided for each bird and therefore received new information about the challenges faced by the declining bird populations in the region. These observations and the resulting codes are specified in Figure 4 below. 


\section{Figure 4}

Inductive themes

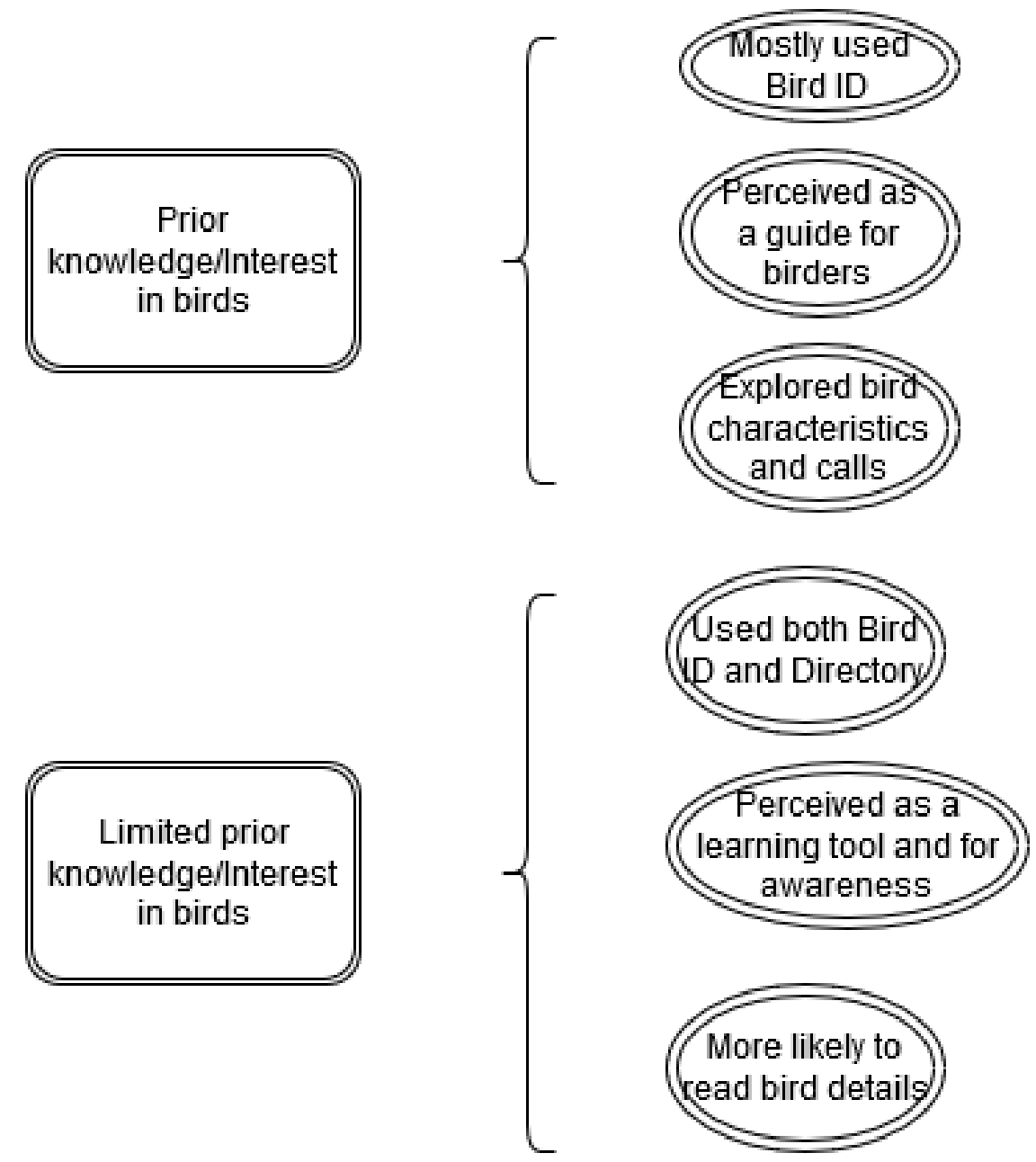

Note. Additional inductive themes and resulting observations

All of the participants suggested some form of a positive experience with using the bird ID app. When probed about awareness, some of the participants had mentioned elements such as water and air pollution, urbanization, and the increased injury to birds during certain festivals such as the Kite Flying festival celebrated in the region. These connections, along with the awareness codes generated in the first cycle of coding allowed the researcher to suggest that 'Gaining information about threats and challenges to birds led to users' having a broader and increased awareness of issues that directly affect birds.'. 
Thematic analysis is often utilized as a methodology that provides flexibility in providing both concept-driven and data driven categories within the coding frame and does not confound by equating the coding frequency of a theme with its importance. Upon completion of the coding frame through an initial pilot analysis of data, a second coding is completed to evaluate and ensure reliability of the coding frame. The coding frame categories are unidimensional and the subcategories are mutually exclusive, meaning any unit can only be coded once in the main category and exhaustive. A directed approach was utilized for the analysis to seek codes that justified or matched the results of the pilot study conducted by the researcher on a similar bird ID app.

\section{Trustworthiness}

This research study attempts to utilize well-accepted qualitative standards while attempting fair treatment of data, as delineated by Guba and Lincoln (1989). The process of utilizing usage statistics to support and complement themes emerging from interviews, and further espousing a directed approach to match results of this study with the results of a prior related pilot study allow for proper rigor within this case study by allowing for triangulation. The emergence and analysis of some of the inductive themes, that were contrary to the reviewed literature or assumptions made by the researcher based upon the results of the pilot study were appropriately acknowledged and discussed. The researcher was also able to confer with at least two of the participants during the process of data analysis to ascertain accuracy of data categorization, thereby using member-checking to improve the trustworthiness. Additionally, the results of the data analysis were discussed with peers and interested experts from the participating institutions, who have shown support for this study and support several conservation related organizations and activities within the general setting of the study 
population. These academic peers and birding enthusiasts also agreed with the results of this study and were supportive of further research and development of the app with additional features to make it more interactive and informational. Some of the suggested features were inclusion of more multimedia elements such as photographs and videos, along with a possible AR experience to be able to better study individual characteristics of each bird in a more realistic virtual environment as well as the addition of educational content pertaining to these birds, such as migratory patterns, breeding seasons, geographical nesting sites. Table 3 outlines the data and analysis specific to constructs that were examined in the current study.

\section{Table 3}

Constructs Examined in the Current Study

\begin{tabular}{llll}
\hline RQs & Constructs Examined & Data Source & Analysis \\
\hline RQ1 & Usage and interactions & $\begin{array}{l}\text { User statistics and history panel of } \\
\text { app }\end{array}$ & $\begin{array}{l}\text { Document } \\
\text { analysis }\end{array}$ \\
\hline RQ2 & Knowledge/awareness & Interviews & $\begin{array}{l}\text { Thematic } \\
\text { analysis }\end{array}$ \\
\hline RQ1 & User Experiences & Comparing codes with pilot study & $\begin{array}{l}\text { Directed } \\
\text { approach } \\
\text { analysis }\end{array}$ \\
\hline
\end{tabular}

\section{Results \& Discussion}

\section{RQ1. Experiences of Users with the Hobby Bird ID Mobile App}

Data analysis suggests that participants had a favorable opinion about the bird ID app as a tool to gain information about local bird life. The themes that emerged during the data analysis indicated a positive overall experience with the app and all of the participants discussed some forms of personally enriching or motivating experiences with the app. This observation of userexperience corroborates a similar user-experience study in the healthcare field, conducted by Lai et al. (2019), which utilized qualitative interviews and presented positive user experience results 
as a collective of participant statements suggesting appreciation, favorable opinions and enthusiasm. As outlined in Figure 3, the participants variously described their experiences as motivational, enriching, convenient, novel, enjoyable or rewarding within their responses and provided several unique use-cases that shed light on how different users' usage and experiences of the app were influenced by several parameters such as use in social settings, usage in urban versus rural settings, use of specific features or use for leisure versus use for seeking specific information.

For instance, two of the participants shared stories about how they had their parents describe certain birds to them, which were no longer commonly seen, and they were able to enter the described characteristics into the app and find the photographs and information about the birds and learn more about the distribution and conservation status of these birds.

Another positive usage instance which came up when asked to discuss unique experiences with the app was when a participant described how they had helped a friend of theirs, who was an avid nature photographer, but did not know the names of the birds that they photographed and shared on their social media accounts, to correctly identify them and provide additional information in their posts. Unique user experience stories provided a nuanced perception on the individual perceptions of the app, for instance, one user discussed connections of the app with their hobby of photography "I would take pictures of birds, but I had no idea what they were, but this app really helped me identify and learn more about the birds that I am seeing". Another participant provided a detailed account of a particular experience with the app: So, there is a specific bird that we see outside our classroom window at my college. I did not know what it was, so I tried to look it up on the app using the bird ID feature and while I found a bird that was similar, it did not look exactly the same. But then I noticed 
the bird call feature in the app and tried it, and this confirmed that it was the same bird. I was very excited.

The flexibility of the semi-structured interviews was extremely useful in encouraging more detailed narrative and allowed the researcher to sequence the interview questions unique to each participant's responses and developing narrative. Another significant observation that emerged during the interviews was that several of the participants had used the app as a tool to learn more about local birds, rather than to ID them, due to a lack of rich birdlife in their immediate urban surroundings. Multiple participants stated that the app was an extremely convenient tool to learn about birds, as compared to books, guides or random searches on the internet. Thus, capturing the unique experiences of the participants, coding these as preliminary and secondary codes, and further analyzing these codes to develop meaningful and insightful themes allowed the researcher to construe that the results of this study supported the findings of the pilot study, and can be reasonably assumed that the sample of college age students who participated in this study had a positive experience with the bird ID mobile app and their general perception about the app, after having used it for a period of time, was that it made them more aware about the local birdlife around them. Doherty et al., (2018) have used similar excerpts from participant interviews to collect data about positive and negative experiences with a pregnancy app.

\section{RQ2. How Do Experiences with the App Influence Knowledge and Awareness of Birdlife?}

Responses from most participants suggest that interaction with the app provided them with some information about local birds that they did not previously possess. Participants who were familiar with birds and had prior interest and knowledge in this area felt that the app gave them more information about bird varieties, categorization, characteristics, calls and habits, 
whereas participants with limited or no prior interest or knowledge of birds had a more wholistic appreciation about the large numbers of birds and the convenient access that the app provided to this new information. Several new themes emerged from the interview data, as outlined in Figure 4, such as the different ways the app was used by the participants leading to unique and individual experiences and stories and the attitudes of participants with prior knowledge of birds towards the app versus the attitudes of participants with limited or no prior knowledge of birds. The difference in the attitudes of users with prior knowledge of birds, as compared to those who did not have previous interest or knowledge provided some deviant observations as compared to the findings from the pilot study and the assumptions made from the review of literature. These users perceived the app as a bird guide for novice birders and tended to use the bird ID feature more commonly, rather than exploring the bird directory feature. They did however acknowledge that they gained new information about birds and found additional information that they were not previously aware of. When the participants with prior knowledge were asked whether the app had provided them with information about birds, beyond what they already knew, one participant stated "Yes, there were several birds that I was not aware of, and I did not even know the names. So that could be useful. You would get new knowledge.”

Other participants with prior knowledge variously made statements such as:

Yes, very definitely. I learned about the specific colors and size characteristics of birds, which is something that we don't always realize. I also learned much about the behavior of different birds. I found this app to be very useful.

Yes of course. I learnt a lot about bird characteristics and the sounds that they make. We often see them, but do not get to hear their calls, so it was interesting to hear the calls of different birds from this app. Yes, I distinctly remember listening several times to the call 
of the Little Gull, as I have not heard it before. This app provides a very convenient and instant access to the information, that is different from books or guides on birds, and I think that this is wonderful. It is very practical.

These participants seemed to focus more on the characteristics, habits and habitats of the birds and did not pay particular attention to information on the conservation status or threats to the birds, listed in the bird app.

Additionally, this study revealed some new findings about the use of the app, and existing as well as developing perceptions of the users about birds. Dennison et al., (2013) noted similar inductive themes emerging during their study on smartphone apps in health behavior change, where participants had differing opinions on the effectiveness of the app for health awareness, based upon their past exposure to such systems. During the interviews, the researcher noted that the participants who had no prior interest and limited knowledge of birds, often confounded wild and domesticated birds. Several of the participants suggested that they could not recall any particular prior experience that they may have had with reference to birds prior to their participation in this study, other than interactions with domesticated birds such as domestic pigeons, parakeets etc. or having seen exotic birds in zoos, as seen in their statements. One of the participants stated "I was not really keen about birds. There are some random incidents where there may have been some white pigeons or caged birds that I have noticed.", while a second participant suggested "My only previous interest in birds is perhaps when I see parrots mimicking on television, and that fascinates me!"

Given the rich birdlife in the region, this observation, not from a single participant, but from several of the participants seems to suggest a lack of overall interest and awareness in wild birds and wildlife among the general population within this age group, and is in complement to 
the arguments presented in the planning of this study. Even the students with some prior knowledge and interest suggested that their interest was very recent and only stemmed from their participation in their institutional birding club and related activities. Moreover, most of these same participants expressed a heightened interest and excitement when they were able to learn about the rich diversity of wild birds in the region from the app, and showed concern upon reading about the rapidly dwindling numbers and the multiple threats and challenges faced by these birds. Participants shared their concerns and ideas in the form of wanting to volunteer, suggesting potential features to be included in the app, such as instructions, tips and emergency contact information for useful resources in the event of bird injuries or related emergencies. At least one participant suggested raising awareness among the farming and pastoral communities due to the heavy impact on birds from the rampant use of pesticides and several of the participants mentioned noticing the decline in the quality of natural water bodies from the irresponsible waste and chemical disposal. These connections between threats to birds and what the participants learned about such threats from the app, and their personal subsequent observations definitely demonstrate increased awareness and sensitivity thereby providing credence and complementing the propositions made by Jhala and Giles (1991) as discussed earlier.

\section{Research Limitations}

The main limitation in this study stems from the limited existing literature on qualitative approaches to user-experience research. This lack of background information inhibits elaborate comparative analysis of results within a directed approach. Furthermore, it provides an opportunity for researcher bias, especially since the researcher has a keen interest in birds and conservation. However, with appropriate cognizance towards rigor and trustworthiness as 
outlined above, the researcher strives to eliminate any possible bias. The researcher does acknowledge that the period of app usage, voluntary download and use of such an app as well as personal initiative to explore such apps may be lower and therefore further research would need to be conducted on multi-faceted approaches to nature education and awareness. Finally, the researcher was not able to study specific user behavior with the app due to geographical limitations. A more thorough study on user behavior and interaction with possible multiple coders for inter-rater reliability, with the app may provide additional knowledge and information in this area.

\section{Conclusion}

A single case study approach is used to explore user experiences with a specific bird ID app and to understand whether such user experiences influence knowledge and awareness. 16 participants from 3 separate participating institutions of higher education in western-India were presented with a specifically designed informational bird ID mobile app. Participant interviews, along with usage statistics were analyzed using coding frames, and the results were further compared with insight obtained from a pilot study performed by the researcher. Research limitations were also discussed.

This study revealed that the participants had a positive experience with the Hobby mobile app and used it in differing ways resulting in unique user experiences. The study further suggested that the app did provide an easy and convenient access to birding and conservation related information and provided the users with knowledge about local birds that influenced their awareness of birds and the challenges and threats towards them. This study therefore serves as a preliminary subjective exploration into possible technology-based interventions to inform and raise awareness about issues of contemporary importance. It contributes to the emerging focus on 
subjective and in-depth exploration of user experience with mobile apps and provides insight for further research and studies in this area. Further studies focused on a mixed methodology may be used to measure the co-relations and provide further triangulation to this format of exploratory research on the impact of mobile apps on knowledge and awareness on specific areas of interest in conservation and other topics. 


\section{References}

Ambastha, K., Hussain, S., \& Badola, R. (2007). Resource dependence and attitudes of local people toward conservation of Kabartal wetland: a case study from the Indo-Gangetic plains. Wetlands Ecology and Management, 15. https://doi.org/10.1007/s11273-006-9029-z

Araz, C. (2018, March 15). Why you should ditch your UX definition, and use this one instead. Retrieved November 25, 2019, from Medium website:

Arts, K., van der Wal, R., \& Adams, W. M. (2015). Digital technology and the conservation of nature. Ambio, 44. https://doi.org/10.1007/s13280-015-0705-1

Communications Today - Internet Usage In Gujarat Higher Than National Average. (n.d.). Retrieved November 4, 2019, from Communications Today website:

https://www.communicationstoday.co.in/internet-usage-in-gujarat-higher-than-national-average/

Creswell, J. W. (1998). Qualitative inquiry and research design: choosing among five traditions. Sage Publications, Inc.

Creswell, J. W., \& Miller, D. L. (2000). Determining Validity in Qualitative Inquiry. Theory Into Practice, 39. https://doi.org/10.1207/s15430421tip3903_2

Creswell, J.W. (2007). Qualitative inquiry and research design: Choosing among five approaches $\left(2^{\text {nd }}\right.$ ed.). Thousand Oaks, CA: Sage

Cuthbert, R. J., Taggart, M. A., Saini, M., Sharma, A., Das, A., Kulkarni, M. D., Green, R. E. (2016). Continuing mortality of vultures in India associated with illegal veterinary use of diclofenac and a potential threat from nimesulide. Oryx, 50. https://doi.org/10.1017/s003060531500037x

Dicicco-Bloom, B., \& Crabtree, B. F. (2006). The qualitative research interview. Medical Education, 40(4), 314-321. 
Dennison, L., Morrison, L., Conway, G., \& Yardley, L. (2013). Opportunities and challenges for smartphone applications in supporting health behavior change: qualitative study. Journal of Medical Internet Research, 15(4), e86.

Doherty, K., Barry, M., Marcano-Belisario, J., Arnaud, B., Morrison, C., Car, J., \& Doherty, G. (2018). A Mobile App for the Self-Report of Psychological Well-Being During Pregnancy (BrightSelf): Qualitative Design Study. JMIR Mental Health, 5(4), e10007.

Einsenhardt, K.M. (1989), Building theories from case study research, Academy of Management Review, 14(4)

Ganpule, P. (2016). The Birds of Gujarat: Status and Distribution. Flamingo, 8(3)-12(4). 2-40.

Garrigan, K. (2014) How Mobile Technology is Changing Conservation | African Wildlife Foundation. Retrieved October 23, 2019, from African Wildlife Foundation website: https://www.awf.org/blog/how-mobile-technology-changing-conservation

Green, R. E., Newton, I., Shultz, S., Cunningham, A. A., Gilbert, M., Pain, D. J., \& Prakash, V. (2004). Diclofenac poisoning as a cause of vulture population declines across the Indian subcontinent. Journal of Applied Ecology, 41. https://doi.org/10.1111/j.0021-8901.2004.00954.x

Guba, E. G., \& Lincoln, Y. S. (1989). Fourth generation evaluation. Newbury Park, CA: Sage

Gustafsson, M., \& Borglin, G. (2013). Can a theory-based educational intervention change nurses’ knowledge and attitudes concerning cancer pain management? A quasi-experimental design. BMC Health Services Research, 13, 328.

Heinen, J. T., \& Shrivastava, R. J. (2009). An analysis of conservation attitudes and awareness around Kaziranga National Park, Assam, India: implications for conservation and development. Population and Environment, 30. https://doi.org/10.1007/s11111-009-0086-0 
Jain, N. (2020, February 21). Most Indian birds declining, finds new report using citizen science data. Mongabay-India. https://india.mongabay.com/2020/02/most-indian-birds-declining-finds-newreport-using-citizen-science-data/

Jhala, Y. V., \& Giles, R. H. (1991). The Status and Conservation of the Wolf in Gujarat and Rajasthan, India. Conservation Biology, 5, https://doi.org/10.1111/j.15231739.1991.tb00354.x

Kim, H., \& Stout, P. A. (2010). The effects of interactivity on information processing and attitude change: implications for mental health stigma. Health Communication, 25(2), 142-154.

Lai, B., Wilroy, J., Young, H.-J., Howell, J., Rimmer, J. H., Mehta, T., \& Thirumalai, M. (2019). A Mobile App to Promote Adapted Exercise and Social Networking for People With Physical Disabilities: Usability Study. JMIR Formative Research, 3(1), e11689.

Marshall, C., \& Rossman, G. B. (1995). Designing qualitative research. Sage Publications, Inc.

Miles, M. B., \& Huberman, A. M. (1994). An expanded sourcebook. Qualitative data analysis (2nd ed.). Thousand Oaks, CA: Sage.

Paik, I.-H., Lim, J., Chun, B.-S., Jin, S.-D., Yu, J.-P., Lee, J.-W., .. Paek, W.-K. (2009). The Korean Bird Information System (KBIS) through open and public participation. BMC Bioinformatics, 10. https://doi.org/10.1186/1471-2105-10-s15-s11

Saldana, J. (2016). The Coding Manual for Qualitative Researchers. SAGE

Stake, R. E. (1995). The Art of Case Study Research. SAGE.

Tang, J., Charles Abraham, Elena Stamp, and Colin Greaves. 2015. "How Can Weight-Loss App Designers’ Best Engage and Support Users? A Qualitative Investigation.” British Journal of Health Psychology 20 (1): 151-71. 
Times of India - In Gujarat, only 42 use internet among every 104 mobile users | Ahmedabad News Times of India. (n.d.). Retrieved November 4, 2019, from The Times of India website: https://timesofindia.indiatimes.com/city/ahmedabad/in-state-only-42-use-internet-among-every104-mobile-users/articleshow/71141657.cms

Trevethan, R. (2017). Deconstructing and Assessing Knowledge and Awareness in Public Health Research. Frontiers in Public Health, 5, 194.

Vaupel, S., Taentzer, G., Gerlach, R., \& Guckert, M. (2018). Model-driven development of mobile applications for Android and iOS supporting role-based app variability. Software \& Systems Modeling, 17, pp. 35-63. https://doi.org/10.1007/s10270-016-0559-4

Vredenburg, K. (2002). Designing the Total User Experience at IBM: An Examination of Case Studies, Research Findings, and Advanced Methods. International Journal of Human-Computer Interaction, 14, https://doi.org/10.1207/s15327590ijhc143\&4_01

VServ. (2013). Retrieved November 25, 2019, from https://www.vserv.com/wpcontent/uploads/2013/09/Mobile-Internet-Consumer-India.pdf

Wieland, L. Susan, Louise Falzon, Chris N. Sciamanna, Kimberlee J. Trudeau, Suzanne Brodney, Joseph E. Schwartz, and Karina W. Davidson. 2012. Interactive Computer-Based Interventions for Weight Loss or Weight Maintenance in Overweight or Obese People. Cochrane Database of Systematic Reviews, no. 8 (August): CD007675.

Yin, Robert K. 2003. Case Study Research: Design and Methods. SAGE. 


\section{Appendix}

Sample interview questions for each of the main research questions:

- RQ1: What are the experiences of college age young adults from western India with the Hobby bird ID mobile app?

○ Tell me how you have used this app.

- Would you like to discuss these specific bird identifications that you have made with the app?

What prompted you to use the app?

- How did you feel when you identified or looked up a bird on the app?

- RQ2: How do these experiences influence their knowledge and awareness of local birdlife?

- Tell me about any early experiences or personal notions/attitudes about birds

○ Discuss any specific observations, interests, experiences about birds

○ Tell me how you feel about birds after having used this app

○ How do you feel about your perception of birds after having used this app?

○ Would you tell me about how this app may have improved your knowledge about birds?

- Are there specific elements such as threats to birds, or decline of birds that you are aware of as a result of this app? Would you please explain? 